\title{
Additional Cervical Lymph Node Biopsy is Not a Significant Prognostic Factor for Nasopharyngeal Carcinoma in the Intensity-Modulated Radiation Therapy Era: A Propensity Score-matched Analysis from an Epidemic Area
}

Xing-Li Yang2* Yan Wang1* Yong Bao ${ }^{*}$, Shao-Bo Liang 3,4 Sha-Sha $\mathrm{He}^{2}$, Dan-Ming Chen ${ }^{1}$, Hai-Yang Chen ${ }^{5}$ Li-Xia Lu², Yong Chen ${ }^{1}$

1. Department of Radiation Oncology, The First Affiliated Hospital of Sun Yat-sen University, Guangzhou, 510060, Guangdong, P.R. China.

2. Department of Radiation Oncology, Sun Yat-sen University Cancer Center.

3. State Key Laboratory of Oncology in South China, Collaborative Innovation Center for Cancer Medicine, Sun Yat-sen University Cancer Center.

4. Department of Radiation Oncology, Cancer Center, First People's Hospital of Foshan Affiliated to Sun Yat-sen University, Foshan, China.

5. The Sixth Affiliated Hospital of Sun Yat-sen University, Guangzhou, China

*These authors contributed equally to this work.

$\square$ Corresponding author: Yong Chen, M.D.; Department of Radiation Oncology, The First Affiliated Hospital of Sun Yat-sen University, Guangzhou, 510060, Guangdong, People's Republic of China. Telephone: +0086-020-87755766 to 8425, E-mail: chenyong@mail.sysu.edu.cn

(1) Ivyspring International Publisher. This is an open access article distributed under the terms of the Creative Commons Attribution (CC BY-NC) license (https:// creativecommons.org/licenses/by-nc/4.0/). See http://ivyspring.com/terms for full terms and conditions.

Received: 2018.02.12; Accepted: 2018.06.09; Published: 2018.07.30

\begin{abstract}
Introduction: This study aimed to evaluate the prognostic value of cervical lymph node biopsy and whether different biopsy methods would lead different outcomes in NPC in the intensity-modulated radiotherapy (IMRT) era.

Material and Methods: 1492 patients with biopsy-proven, non-metastatic NPC, and treated by IMRT with or without chemotherapy were retrospectively reviewed. Cervical lymph node biopsy was performed in 183 (12.3\%) patients: $61(4.1 \%)$ by needle puncture and $118(7.9 \%)$ by excision biopsy. Propensity-score matching was used to match patients in both arms at an equal ratio. Overall survival (OS), distant metastasis-free survival (DMFS), locoregional relapse-free survival (LRFS), and nodal relapse-free survival (NRFS) were assessed using the Kaplan-Meier method and compared using the log-rank test. Independent prognostic factors were identified using the Cox proportional hazards model.

Results: In the original cohort of 1492 patients, patients receiving cervical lymph node biopsy had comparable survival (OS: $P=0.736$, DMFS: $P=0.749$, LRFS: $P=0.538$, NRFS: $P=0.093$,) with patients receiving isolated napharynx biopsy. The results for the propensity-match cohort of 316 patients were similar. Interestingly, compared with the control group and needle puncture biopsy group, a slightly lower nodal recurrence rate was observed in the excision biopsy group $(P=0.082$ and $P=0.072$, respectively). Adjusting for the known prognostic factors in multivariate analysis, cervical biopsy did not cause a higher risk of death, distant metastasis, or nodal relapse.
\end{abstract}

Conclusions: Pretreatment cervical lymph node biopsy is not associated with impaired survival in NPC, suggesting the resist of the biopsy and more aggressive treatment after the biopsy may be unnecessary.

Key words: nasopharyngeal carcinoma, intensity-modulated radiotherapy, cervical lymph nodes biopsy, prognosis.

\section{Introduction}

Nasopharyngeal carcinoma (NPC) is a unique malignancy with marked racial and geographical differences. Compared with an incidence in the United States of 0.5 to 2 per 100,000 [1], the crude 
incidence of NPC in China was 3.09/100,000 in 2012 [2]. Notably, the highest incidence was found in South China, which was 3.4 times higher than the second highest area - Southwest China.

Patients with NPC may remain asymptomatic for a long time, and 3-5\% of patients present with a neck mass when primary symptoms are absent $[3,4]$. Conventional tests, such as fiber optic examination and biopsy of the primary site, were recommend for diagnosis in previous National Comprehensive Cancer Network (NCCN) guidelines [5]. In the latest guidelines [6], fine-needle aspiration biopsy (FNAB) of the neck was added. However, neck biopsy was not recommended in the European Society for Medical Oncology (ESMO)-European Society for Radiotherapy \& Oncology (ESTRO) Clinical Practice Guideline for NPC in 2012 [7].

Previous studies regarding lymph node biopsy in NPC also reported different outcomes. In the last century, a study from North America [8] asserted that pre-irradiation node biopsy might result in poorer survival; however, another study, mainly based on a Chinese population [9], stated the oppose opinion. Since intensity-modulated radiotherapy (IMRT) entered clinical practice at the end of the last century, great improvements in survival have been achieved $[10,11]$. Moreover, the addition of chemotherapy also reduced the mortality of NPC [12]. IMRT is the current standard of treatment for NPC, and additional chemotherapy has been applied widely to patients in the advance stage. However, it remains unknown whether the addition of cervical lymph nodes biopsy might change the chance of survival in IMRT era. Recently a population-based study [13] concluded that pretreatment neck biopsy was not associated with lower overall survival but in differentiated non-keratinizing carcinoma group, lymph node biopsy had an independent adverse effect on survival. Nevertheless, as a propensity-score-match study, the data failed to assess some important elements, including EBV-DNA, chemotherapeutic regimens, radiotherapy techniques, and radiation dose.

Moreover, NPC is a solid tumor with no symptoms except for a neck mass, which is easy to confuse with lymphoma; therefore, lymph node excision biopsy is the main method of neck biopsy in NPC. Image-guided needle biopsy has been recommended by some authors for diagnosis because of its comparable accuracy, higher tolerability, better economic applicability, and fewer post-procedural complications and delays [14, 15]. However, few of these studies investigated the prognostic value of different biopsy methods. Therefore, whether needle biopsy or excision biopsy are associated with different prognoses requires further discussion.
This study aimed to verify the impact of cervical lymph node biopsy and further investigate whether different biopsy methods would lead to different outcomes of NPC in the IMRT era.

\section{Methods and Materials}

\section{Patient characteristics}

A cohort of 1492 patient with NPC who were treated at Sun Yat-sen University Cancer Center with IMRT between January 2010 and September 2013 were retrospectively reviewed. All the patients were newly diagnosed as non-metastatic NPC with biopsy and magnetic resonance imaging (MRI) of nasopharynx and neck, chest radiography or computed tomography (CT), abdominal sonography or CT, and technetium-99m-methylene diphosphonate wholebody bone scan, and/or [18F] fluorodeoxyglucose positron emission tomography and CT. Patients were retrospectively re-staged according to the $8^{\text {th }}$ edition of UICC/AJCC staging system.

\section{Treatment}

IMRT were delivered as the primary treatment to the patients once daily with five fractions per week. Prescribed radiation doses were 68-70 Gy in 30-33 fractions at 2.13-2.27 Gy/fraction to the planning target volume (PTV) of GTV-P, 60 Gy to the PTV of CTV-1 (high-risk regions), 54 Gy to the PTV of CTV-2 (low-risk regions and neck nodal regions), and 60-68 Gy to the nodal gross tumor volume (GTV-N) in 30-33 fractions.

According to the national comprehensive cancer network (NCCN) guidelines, platinum-based chemotherapy was delivered to $1254(84.0 \%)$ patients with stage II-IVA disease: the sequence given was induction chemotherapy alone $176(11.7 \%)$, concurrent chemotherapy alone 436(29.2\%), adjuvant chemotherapy alone $2(0.01 \%)$, induction-concurrent chemotherapy $572(38.3 \%)$, concurrent-adjuvant chemotherapy $6(0.03 \%)$, induction-adjuvant chemotherapy $2(0.01 \%)$, induction-concurrent-adjuvant chemotherapy $5(0.03 \%)$.

Patients' refusal, age ( $\geq 70$ years), organ severe dysfunction (diabetes, cardiac dysfunction, renal insufficiency, liver insufficiency, et al) that would suggest intolerance to chemotherapy may led to deviation from institutional guidelines. IC regimen consisted of docetaxel/ paclitaxel plus cisplatin/ nedaplatin, cisplatin/nedaplatin plus fluorouracil, or docetaxel/paclitaxel plus cisplatin/nedaplatin plus fluorouracil given every 3 weeks for 2 to 3 cycles before radiotherapy. CC regimen consisted of 80 to $100 \mathrm{mg} / \mathrm{m} 2$ cisplatin given every 3 weeks for 2 to 3 cycles, or 30 to $40 \mathrm{mg} / \mathrm{m} 2$ cisplatin given weekly for up to 7 cycles. 


\section{Follow-up}

Patients received routine follow up every 3 to 6 months during the first 3 years and every 6 to 12 months thereafter. Telephone follow-up was delivered to those without recent examination tests in the medical records. The overall survival (OS), local relapse-free survival (LRFS), regional relapse-free survival (RRFS), distant metastasis-free survival (DMFS), and progression-free survival (PFS) were calculated from the day of first diagnosis to death or last follow-up (November $8^{\text {th }}, 2017$ ), local failure, regional failure, and distant failure, respectively.

\section{Statistical analysis}

The SPSS 22.0 software package (SPSS Inc., Chicago, IL) was applied for all statistical analyses. Baseline characteristics were compared between groups using the $x^{2}$ test (categorical variable), and standardized difference. Survival analyses were conducted using the Kaplan-Meier method, and the log-rank test was used to compare the differences.

This study calibrated selection bias and confounding bias using PSM for randomization. The original data were used for logistic regression to calculate propensity scores in the control group and treatment group.

In our research, the two groups were divided based on the receipt of cervical lymph node biopsy by 1:1 matching with the following matching factors: gender (male vs. female), age ( $\leq 45$ years $v s$. $>45$ years), histological type (I-II and III), smoke history (yes vs. no), T category (T1-2 vs. T3-4), N category (N0-1 vs. N2-3), clinical stage (I-II vs. III-IVa), concurrent chemotherapy (yes vs. no), induction chemotherapy (yes vs. no), pre-treatment EBV DNA ( $\leq$ $4,000$ vs. $>4,000)$. A P value of less than 0.05 was considered statistically significant.

\section{Result}

\section{Patients}

Initially, $1309(87.7 \%)$ patients receive isolated biopsy of primary site (control arm) and 183(12.3\%) patients received both nasopharynx and cervical lymph node biopsy (biopsy arm). Of note, 61(4.1\%) patients received lymph node biopsy group by fine-needle aspiration biopsy (FNAB), and 118 (7.9\%) by excision biopsy, which was complete remove of a single lymph node, the other 4 patients had equivocal biopsy. Following propensity score matching, nasopharyngeal biopsy was delivered to 338 patients, and half of them received additionally biopsy of the neck. The matched patients in both arms had balanced characteristics (Table 1).
Table 1. Clinical characteristics of 1492 patients before propensity score matching (PSM) and 316 patients after PSM

\begin{tabular}{|c|c|c|c|c|c|c|}
\hline \multirow[t]{2}{*}{ Factora } & \multicolumn{3}{|c|}{ All case } & \multicolumn{3}{|c|}{ Match case } \\
\hline & $-e$ & $+f$ & $P$ & - & + & $P$ \\
\hline Gender & & & 0.751 & & & 1.000 \\
\hline Male & 1065 & 145 & & 125 & 126 & \\
\hline Female & 244 & 78 & & 33 & 32 & \\
\hline Age & & & 0.500 & & & 0.698 \\
\hline$\leq 45$ & 880 & 128 & & 116 & 120 & \\
\hline$>45$ & 429 & 55 & & 42 & 38 & \\
\hline Histology b & & & 0.453 & & & 1 \\
\hline Type 1 or 2 & 63 & 6 & & 5 & 4 & \\
\hline Type 3 & 1246 & 177 & & 153 & 154 & \\
\hline Smoke & & & 1.000 & & & 1 \\
\hline No & 891 & 125 & & 112 & 113 & \\
\hline Yes & 418 & 58 & & 46 & 45 & \\
\hline $\begin{array}{l}\text { Induction } \\
\text { Chemotherapy }\end{array}$ & & & 0.001 & & & 0.907 \\
\hline No & 620 & 63 & & 58 & 60 & \\
\hline Yes & 689 & 120 & & 100 & 98 & \\
\hline $\begin{array}{l}\text { Concurrent } \\
\text { Chemotherapy }\end{array}$ & & & 0.927 & & & 1 \\
\hline No & 316 & 45 & & 27 & 27 & \\
\hline Yes & 993 & 138 & & 131 & 131 & \\
\hline EBV DNA ${ }^{c}$ & & & 0.751 & & & 0.734 \\
\hline$<4000$ & 734 & 105 & & 86 & 90 & \\
\hline$\geq 4000$ & 575 & 78 & & 72 & 68 & \\
\hline Year & & & 0.256 & & & 0.649 \\
\hline $2010-2011$ & 492 & 77 & & 69 & 64 & \\
\hline $2012-2013$ & 817 & 106 & & 89 & 94 & \\
\hline T-classification d & & & $<0.001$ & & & 0.910 \\
\hline $1 \sim 2$ & 460 & 105 & & 83 & 85 & \\
\hline $3 \sim 4$ & 849 & 78 & & 75 & 73 & \\
\hline N-classification d & & & 0.003 & & & 1 \\
\hline $0 \sim 1$ & 975 & 117 & & 105 & 106 & \\
\hline $2 \sim 3$ & 334 & 66 & & 53 & 52 & \\
\hline Clinical stage $^{\mathrm{d}}$ & & & 0.008 & & & 0.818 \\
\hline $\mathrm{I} \sim \mathrm{II}$ & 385 & 72 & & 60 & 63 & \\
\hline III $\sim \mathrm{IV}$ & 924 & 111 & & 98 & 95 & \\
\hline
\end{tabular}

a: All the factors was analysed using $\times 2$ test (categorical variable)

b:Histology was based on the criteria of WHO histological type (1991): type 1 (keratinizing squamous cell carcinoma), type 2 (differentiated non-keratinizing carcinoma), type 3 (undifferentiated non-keratinizing carcinoma), and other

(carcinomas not otherwise specified)

c: The boundary was based on the previous study from the same center.

$\mathrm{d}$ : According to the $8^{\text {th }}$ edition of the American Joint Committee on Cancer (AJCC) stage classification

e: Without lymph node biopsy.

f: With lymph node biopsy.

\section{Survival Outcome}

In the primary unmatched cohort of 1492 patients, median follow-up time was 57.1 months (0.9-93.9 months) for the control arm and 56.2 months (2.5-83.3 months) for the biopsy arm, respectively. Overall, 4-year OS, DMFS, LRFS and NRFS rates did not differ significantly between the two arms (OS $91.3 \%$ vs. $91.0 \%, P=0.736$; DMFS $85.4 \%$ vs. $85.5 \%, P=$ 0.749; LRFS $94.3 \%$ vs. $97.6 \%, P=0.538$; and NRFS $93.8 \%$ vs. $98.2 \%, P=0.093$; Figure $1, \mathrm{~A}-\mathrm{D})$.

In the propensity-matched cohort of 326 patients, median follow-up time was 61.5 months (2.7-75.5 months) for the control arm and 56.5 months (2.5-83.3 months) for the biopsy arm, respectively. In univariate analysis, IC plus IMRT achieved similar survival to IMRT plus CC (4-year OS $88.4 \%$ vs. $92.4 \%$, $P=0.195$; DMFS $85.8 \%$ vs. $86.5 \%, P=0.579$; LRFS 
A

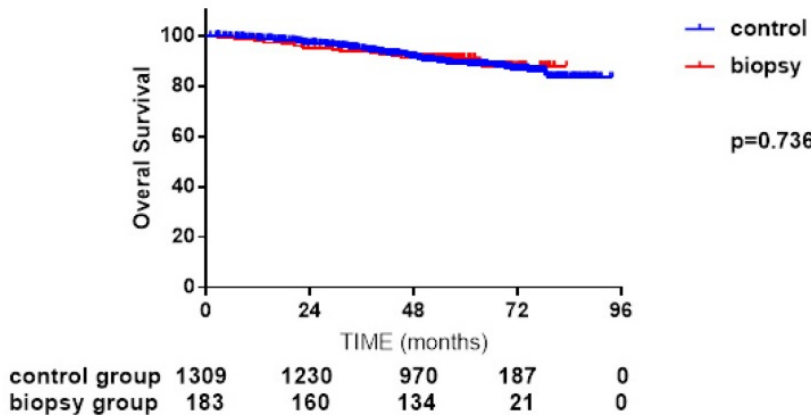

C

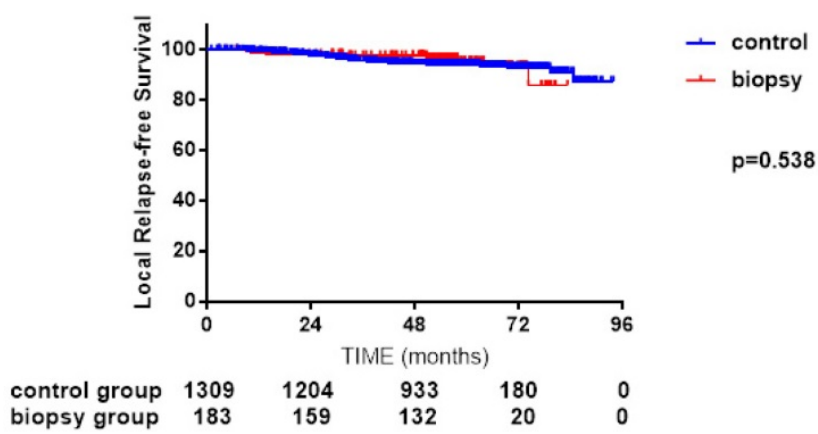

B

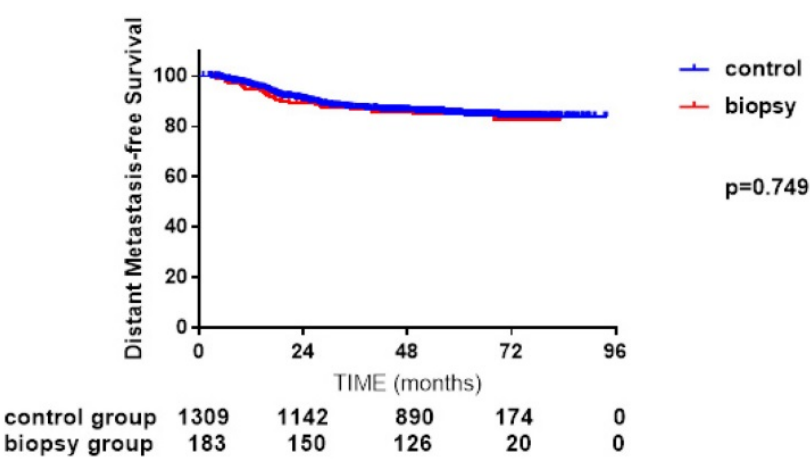

D

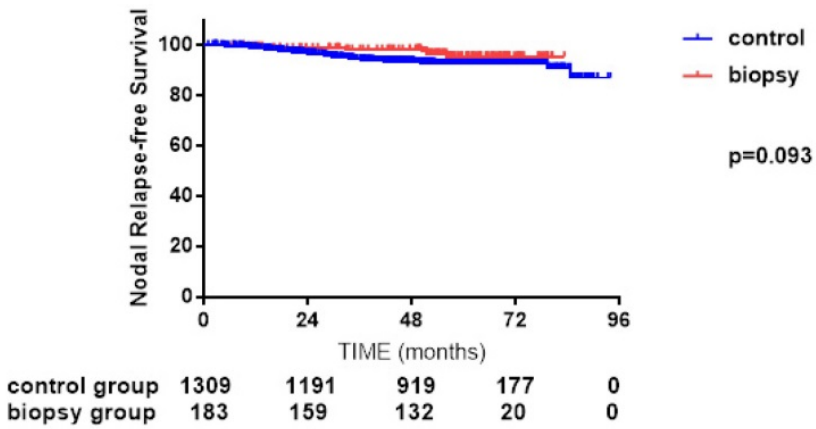

Figure 1. Comparisons of overall survival (A), distant metastasis-free survival (B), local relapse-free survival (C) and nodal relapse-free survival (D) curves between 183 NPC patients with cervical lymph node biopsy and 1309 NPC patients without cervical lymph node biopsy.

93.9\% vs. $97.3 \%, P=0.565$; and NRFS $94.4 \%$ vs. $97.9 \%$, $P=0.204 ;$ Figure 2, A-D).

In the cervical lymph node biopsy cohort of 183 patients, median follow-up tome was 54.4 months (2.5-79.0 months) for FNAB group and 56.6 months (4.5-83.3 months) for excision biopsy group. Marginally significant was found in NRFS between these two groups but other survivals were highly similar. (4-year OS $90.6 \%$ vs. $93.2 \%, P=0.826$; DMFS $87.6 \%$ vs. $84.4 \%, P=0.601$; LRFS $98.2 \%$ vs. $96.4 \%, P$ $=0.203$ and NRFS $99.1 \%$ vs. $96.4 \%, P=0.082$; Figure 3 , A-D).

We conducted a subgroup analysis to investigate how different biopsy ways influenced the pattern of failure and survival. All covariates were well balanced in each stratum between the biopsy and non-biopsy groups by propensity score-matching. No significant difference was found between needle biopsy group and non-biopsy group (Figure 4, A-D), excision biopsy group and non-biopsy group (Figure 5, A-D). Interestingly, excision biopsy group presented marginally lower nodal recurrence $(P=$ 0.072 ).
Accounting for age (categorical), sex, clinical stage, pretreatment EBV DNA, chemotherapy in multivariate analysis, cervical lymph node excision biopsy failed to associate with higher risk of death, distant metastasis, local or regional relapse than other patients (received isolated nasopharyngeal biopsy and received additional fine-needle aspiration biopsy) (Table 2).

Table 2. Summary of multivariate analysis of prognostic factors in 1492 nasopharyngeal carcinoma patients.

\begin{tabular}{llllll}
\hline Endpoint & Variable & $\mathrm{B}$ & $P$ & $\mathrm{HR}$ & 95\% CI For HR \\
\hline OSa & EBV DNA & 0.455 & 0.007 & 1.577 & $1.134-2.192$ \\
& Age $(\leq 45$ vs. $>$ & 0.521 & 0.001 & 1.683 & $1.227-2.309$ \\
& 45) & & & & \\
& N-classification & 0.855 & $<0.001$ & 2.351 & $1.668-3.312$ \\
& Clinical stage & 0.749 & 0.005 & 2.114 & $1.257-3.556$ \\
DMFS b & EBV DNA & 0.657 & $<0.001$ & 1.928 & $1.451-2.562$ \\
& Age ( $\leq 45$ vs. $>$ & 0.299 & 0.033 & 1.348 & $1.024-1.776$ \\
& 45) & & & & \\
& N-classification & 0.963 & $<0.001$ & 2.621 & $1.989-3.453$ \\
NRFSc & Clinical stage & 0.515 & 0.044 & 1.673 & $1.015-2.759$ \\
\hline
\end{tabular}

a: overall survival

b: distant metastasis-free survival

c: nodal relapse-free survival 
A

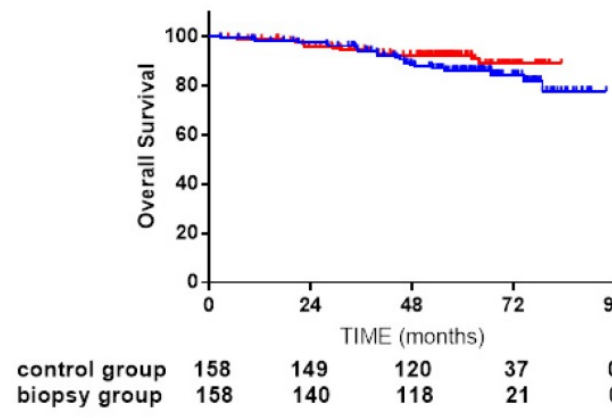

C

4LRFS

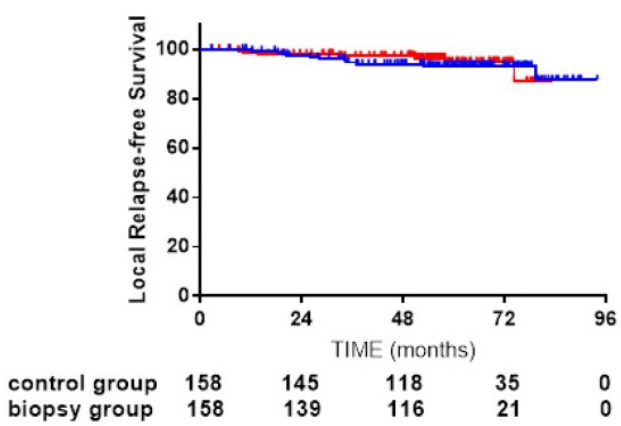

B
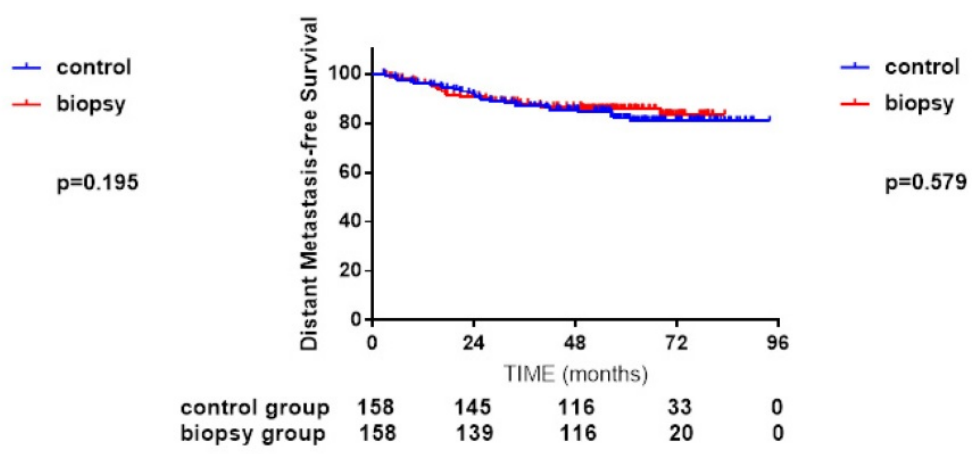

D
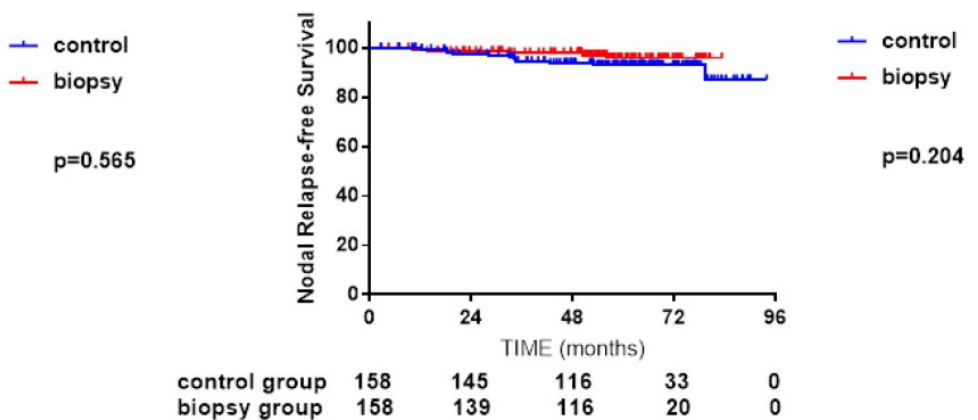

Figure 2. Comparisons of overall survival (A), distant metastasis-free survival (B), local relapse-free survival (C) and nodal relapse-free survival (D) curves between 158 NPC patients with cervical lymph node biopsy and 158 NPC patients without cervical lymph node biopsy after propensity score matching (PSM).

A

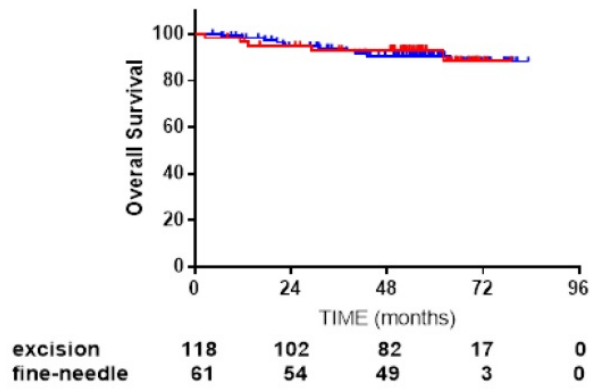

C

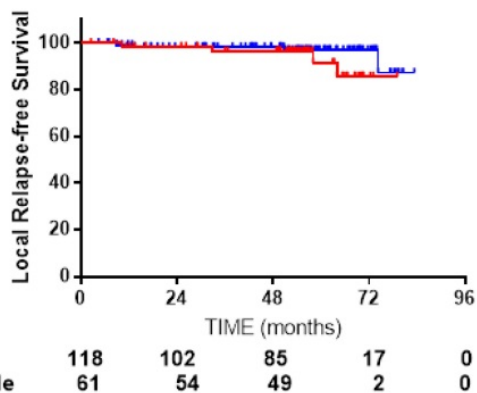

B
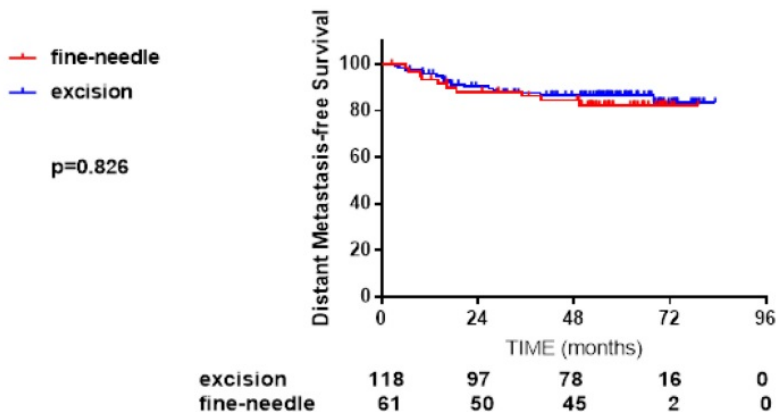

D

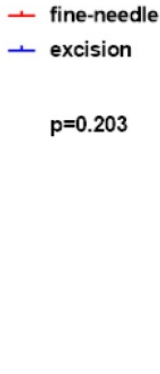

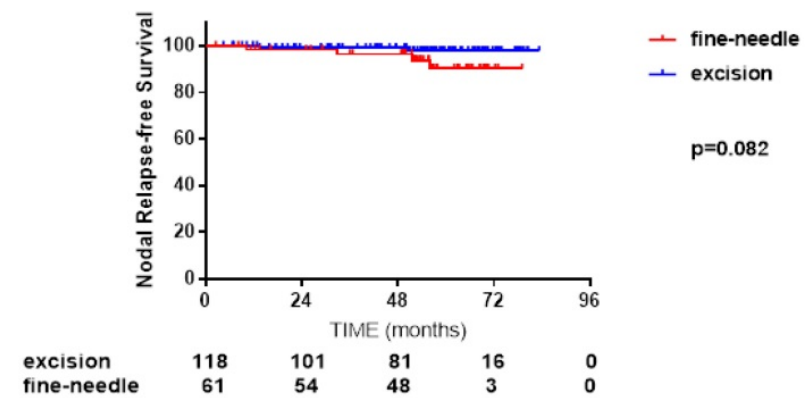

+ fine-needle

+ excision

$p=0.601$

Figure 3. Comparisons of overall survival (A), distant metastasis-free survival (B), local relapse-free survival (C) and nodal relapse-free survival (D) curves between 118 NPC patients received cervical lymph node fine-needle biopsy and 61 NPC patients received cervical lymph node excision biopsy. 
A

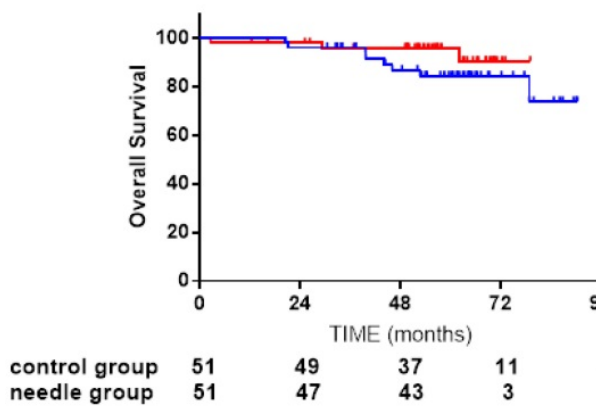

C

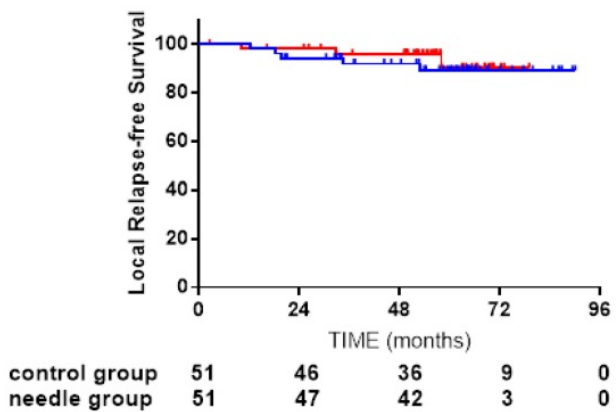

B

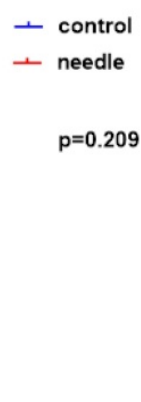

- control

$\leftarrow$ needle

$p=0.562$
- control

$\leftarrow$ needle

$p=0.437$

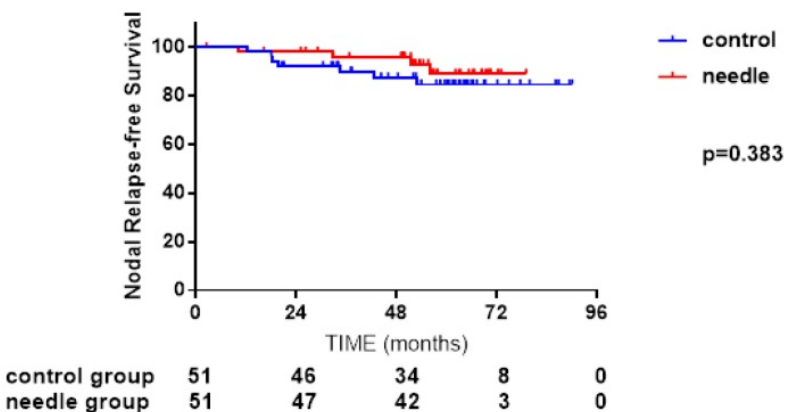

- control

- needle

$p=0.383$

$\begin{array}{llllll}\text { control group } & 51 & 46 & 34 & 9 & 0 \\ \text { needle group } & 51 & 44 & 41 & 3 & 0\end{array}$

D

Figure 4. Comparisons of overall survival (A), distant metastasis-free survival (B), local relapse-free survival (C) and nodal relapse-free survival (D) curves between 51 NPC patients with cervical lymph node needle biopsy and 51 NPC patients without cervical lymph node biopsy after propensity score matching (PSM).

A

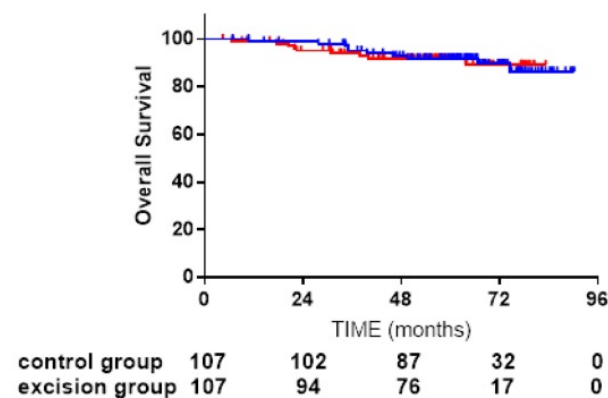

C

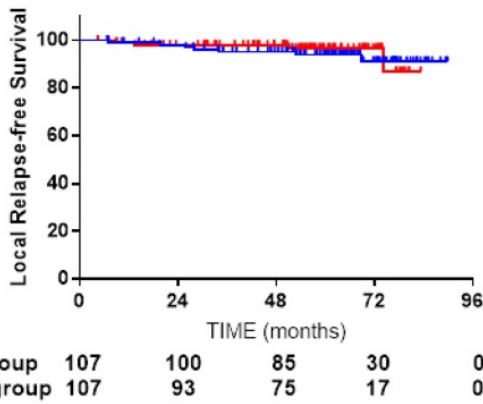

B
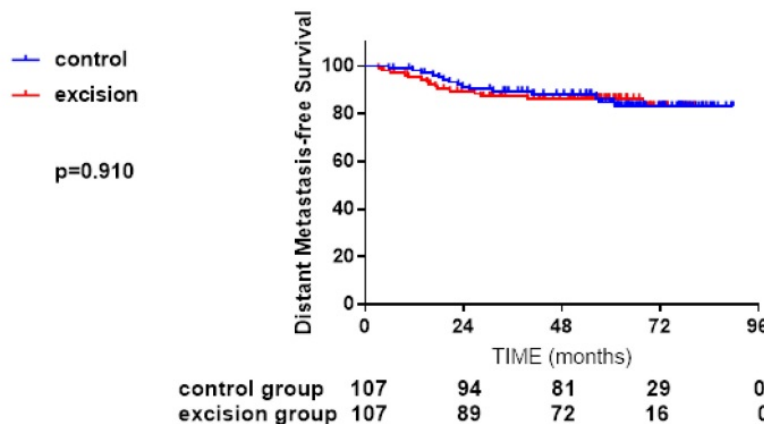

+ control

+ excision

$p=0.939$
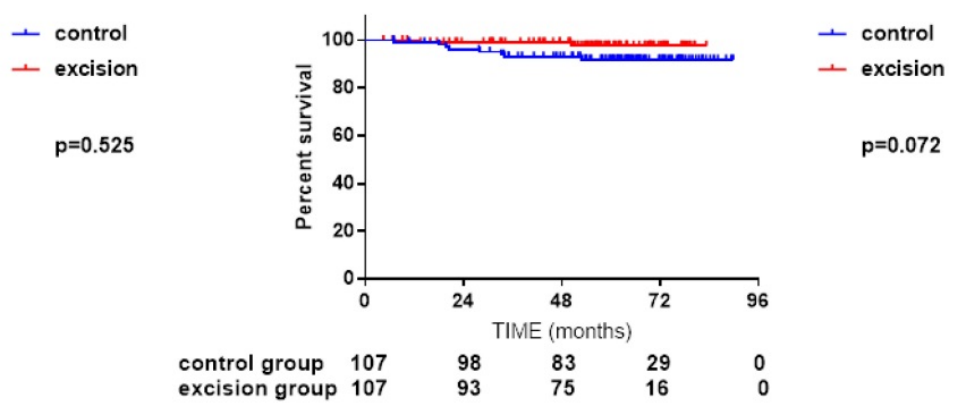

Figure 5. Comparisons of overall survival (A), distant metastasis-free survival (B), local relapse-free survival (C) and nodal relapse-free survival (D) curves between $107 \mathrm{NPC}$ patients with cervical lymph node excision biopsy and 107 NPC patients without cervical lymph node biopsy after propensity score matching (PSM) 


\section{Discussion}

For unknown primary malignancy with neck nodes metastasis, cervical lymph node biopsy is an important diagnostic method. FNAB of the neck was added as a recommended test to diagnose NPC in latest National Comprehensive Cancer Network (NCCN) guidelines [6]. However, misgivings over the promotion of tumor cell dissemination led to a different recommendation in the ESTRO Clinical Practice Guidelines for NPC in 2012 [7]. A population-based study showed that no significant impaired survival was found in neck biopsy of NPC; however, the study failed to discuss the method of biopsy in detail [13]. To date, only one, relatively old article stated that partial excision presented poorer survival than complete excision [9]. However, in the study cohort, the treatment received by the patients varied, which decreased the reliability of the results. Currently, IMRT is the therapeutic benchmark for NPC [16]; thus, a study performed before the advent of IMRT may no longer be relevant.

Therefore, in the present study, we conducted a propensity score-matched assessment of the impact of cervical lymph node biopsy in NPC in the IMRT era.

In our study, biopsy of neck nodes did not result in a higher incidence of death, which supported the NCCN guidelines and Lv's study [13]. In fact, studies in 1981 [8] and 1983 [9] also found no significant survival difference between two group. However, the article by Dickson [8] et al supposed that the trend was unmistakable when $39 \%$ of 58 biopsied patients and $31 \%$ of 50 non-biopsied patients developed distant metastasis. Although there were concerns about tumor cell dissemination and formation of micro-metastatic foci caused by biopsy [17-19], our study showed that neck biopsy would not increase the risk of nodal recurrence or distant metastasis. One reasonable conjecture was that the "impairment" resulting from cervical lymph node biopsy was curable for NPC. Chemotherapy was supposed to markedly reduced the risk of metastasis and improve radiosensitivity [12], which was recommended for positive lymph node metastasis in the national practice guidelines [7]. Meanwhile, the widespread application of MRI and IMRT remarkably improved the survival, especially locoregional recurrence-free survival, of patients with NPC [11, 20]. In other words, distant metastasis is the main treatment failure pattern and is associated with poorer with overall survival. As cervical node biopsy would not result in impaired DMFS or OS, more aggressive treatment may not necessary.

Interestingly, in the subgroup analysis, lower nodal recurrence in patients who received pretreatment nodal excision biopsy was marginally significant. We noticed that some oncologists treated the area of the biopsied node as "tumor bed" and a higher dose of radiation was usually delivered to neck, leading to a lower nodal recurrence rate in node-biopsy group. However, the highly similar overall survival curve between the excision biopsy and control groups suggested that the higher dose was unnecessary.

Although only FNAB was recommended as a diagnostic test in head and neck cancer, surgical excision biopsy was considered ideal for diagnosis when NPC was misdiagnosed as lymphoma [21]. However, the expense, the potential for treatment delay, and the low compliance because of the surgical approach call for another, less invasive method of tissue sampling. Cai et al found patients who received radiotherapy within 14 days after biopsy had a five-year survival of $61 \%(42 / 69)$, which is better than that of the patients who started their treatment beyond the $15^{\text {th }}$ day $(47.5 \% \sim 58 / 122)[9]$. Imagineguided core needle biopsy has been proven to provide sufficient information and minimal impairment compared with excision [14], whereas FNAB was accused of being "woefully inadequate" for treatment planning [22]. Fortunately, in contrast to lymphoma, lymph node biopsy is secondary in NPC diagnosis and the nodal architecture plays a minor role. In addition, we found that cervical needle biopsy was not associated with significant survival impairment. Therefore, the recommendation to use FNAB to diagnose NPC is feasible and safe.

As a retrospective study from a single center in an epidemic area, our results may be limited by selection bias. Second, as most of neck biopsies were done by other centers, it was hard to obtain the details of the biopsied nodes, such as their volume [23], extracapsular extension [24], and necrosis [25], which are believed to be crucial factors for treatment induction and prognosis. Third, the lack of details concerning the actual radiation dose distribution might affect the creditability of results. Moreover, the single race and histological type may limit the application of this study.

\section{Conclusion}

In the IMRT era, additional cervical lymph node biopsy would not increase the risk of local or distant failure, or death. For the excision-biopsy cohort, lower nodal recurrence did not result in increased survival. Therefore, cervical lymph node biopsy could be added as conventional diagnostic test for NPC and more aggressive chemotherapy or a higher radiation dose to neck for patients who received node excision biopsy may not be necessary. 


\section{Acknowledgments}

This work was supported by the Natural Science Foundation of Guangdong Province, China [grant number 2016A020215083]; Medical Science and Technology Research Foundation of Guangdong Province, China [grant number A02016031]. The funding agency had no role in the study design, data collection and analysis, decision to publish, or preparation of the manuscript.

\section{Competing Interests}

The authors have declared that no competing interest exists.

\section{References}

1. Chang ET, Adami HO. The enigmatic epidemiology of nasopharyngeal carcinoma. Cancer epidemiology, biomarkers \& prevention : a publication of the American Association for Cancer Research, cosponsored by the American Society of Preventive Oncology. 2006; 15: 1765-77.

2. Wei KR, Zheng RS, Zhang SW, Liang ZH, Li ZM, Chen WQ. Nasopharyngeal carcinoma incidence and mortality in China, 2013. Chinese journal of cancer. 2017; 36: 90.

3. Grau C, Johansen LV, Jakobsen J, Geertsen P, Andersen E, Jensen BB. Cervical lymph node metastases from unknown primary tumours. Results from a national survey by the Danish Society for Head and Neck Oncology. Radiotherapy and oncology : journal of the European Society for Therapeutic Radiology and Oncology. 2000; 55: 121-9.

4. Tong CC, Luk MY, Chow SM, Ngan KC, Lau WH. Cervical nodal metastases from occult primary: undifferentiated carcinoma versus squamous cell carcinoma. Head \& neck. 2002; 24: 361-9.

5. Pfister DG, Spencer S, Brizel DM, Burtness B, Busse PM, Caudell JJ, et al. Head and neck cancers, Version 2.2014. Clinical practice guidelines in oncology. Journal of the National Comprehensive Cancer Network : JNCCN. 2014; 12: 1454-87.

6. Adelstein D, Gillison ML, Pfister DG, Spencer S, Adkins D, Brizel DM, et al. NCCN Guidelines Insights: Head and Neck Cancers, Version 2.2017. Journal of the National Comprehensive Cancer Network : JNCCN. 2017; 15: 761-70.

7. Chan AT, Gregoire V, Lefebvre JL, Licitra L, Hui EP, Leung SF, et al. Nasopharyngeal cancer: EHNS-ESMO-ESTRO Clinical Practice Guidelines for diagnosis, treatment and follow-up. Annals of oncology : official journal of the European Society for Medical Oncology. 2012; 23 Suppl 7: vii83-5.

8. Dickson RI. Nasopharyngeal carcinoma: an evaluation of 209 patients. The Laryngoscope. 1981; 91: 333-54.

9. Cai WM, Zhang HX, Hu YH, Gu XZ. Influence of biopsy on the prognosis of nasopharyngeal carcinoma--a critical study of biopsy from the nasopharynx and cervical lymph node of 649 patients. International journal of radiation oncology, biology, physics. 1983; 9: 1439-44.

10. Chao KS, Low DA, Perez CA, Purdy JA. Intensity-modulated radiation therapy in head and neck cancers: The Mallinckrodt experience. International journal of cancer. 2000; 90: 92-103.

11. Zhang MX, Li J, Shen GP, Zou X, Xu JJ, Jiang R, et al. Intensity-modulated radiotherapy prolongs the survival of patients with nasopharyngeal carcinoma compared with conventional two-dimensional radiotherapy: A 10 -year experience with a large cohort and long follow-up. European journal of cancer (Oxford, England : 1990). 2015; 51: 2587-95.

12. Blanchard P, Lee A, Marguet S, Leclercq J, Ng WT, Ma J, et al. Chemotherapy and radiotherapy in nasopharyngeal carcinoma: an update of the MAC-NPC meta-analysis. The Lancet Oncology. 2015; 16: 645-55.

13. Lv JW, Zhou GQ, Chen YP, Tang LL, Mao YP, Chen L, et al. Refining the Role of Lymph Node Biopsy in Survival for Patients with Nasopharyngeal Carcinoma: Population-Based Study from the Surveillance Epidemiology and End-Results Registry. Annals of surgical oncology. 2017; 24: 2580-7.

14. Skelton E, Jewison A, Okpaluba C, Sallomi I, Lowe J, Ramesar K, et al Image-guided core needle biopsy in the diagnosis of malignant lymphoma. European journal of surgical oncology : the journal of the European Society of Surgical Oncology and the British Association of Surgical Oncology. 2015; 41: 852-8.

15. Pugliese N, Di Perna M, Cozzolino I, Ciancia G, Pettinato G, Zeppa P, et al. Randomized comparison of power Doppler ultrasonography-guided core-needle biopsy with open surgical biopsy for the characterization of lymphadenopathies in patients with suspected lymphoma. Annals of hematology. 2017; 96: 627-37.

16. Lee AW, Ng WT, Chan LL, Hung WM, Chan CC, Sze HC, et al. Evolution of treatment for nasopharyngeal cancer--success and setback in the intensity-modulated radiotherapy era. Radiotherapy and oncology : journal of the European Society for Therapeutic Radiology and Oncology. 2014; 110: 377-84.

17. Heimbach JK, Sanchez W, Rosen CB, Gores GJ. Trans-peritoneal fine needle aspiration biopsy of hilar cholangiocarcinoma is associated with disease dissemination. HPB : the official journal of the International Hepato Pancreato Biliary Association. 2011; 13: 356-60.

18. AlHilli MM, Podratz KC, Dowdy SC, Bakkum-Gamez JN, Weaver AL, McGree $\mathrm{ME}$, et al. Preoperative biopsy and intraoperative tumor diameter predict lymph node dissemination in endometrial cancer. Gynecologic oncology. 2013; 128: 294-9.

19. Young AL, Lodge JP. Needle-track seeding following biopsy of liver lesions in the diagnosis of hepatocellular cancer: a systematic review and meta-analysis. Gut. 2009; 58: 887-8.

20. Liao XB, Mao YP, Liu LZ, Tang LL, Sun Y, Wang Y, et al. How does magnetic resonance imaging influence staging according to AJCC staging system for nasopharyngeal carcinoma compared with computed tomography? International journal of radiation oncology, biology, physics. 2008; 72: 1368-77.

21. Tilly H, Gomes da Silva M, Vitolo U, Jack A, Meignan M, Lopez-Guillermo A, et al. Diffuse large B-cell lymphoma (DLBCL): ESMO Clinical Practice Guidelines for diagnosis, treatment and follow-up. Annals of oncology : official journal of the European Society for Medical Oncology. 2015; 26 Suppl 5: v116-25.

22. Hehn ST, Grogan TM, Miller TP. Utility of fine-needle aspiration as a diagnostic technique in lymphoma. Journal of clinical oncology : official journal of the American Society of Clinical Oncology. 2004; 22: 3046-52.

23. Liang SB, Teng JJ, Hu XF, Yang XL, Luo M, Fang XN, et al. Prognostic value of total tumor volume in patients with nasopharyngeal carcinoma treated with intensity-modulated radiotherapy. BMC cancer. 2017; 17: 506.

24. Sinha P, Lewis JS, Jr., Piccirillo JF, Kallogjeri D, Haughey BH. Extracapsular spread and adjuvant therapy in human papillomavirus-related, p16-positive oropharyngeal carcinoma. Cancer. 2012; 118: 3519-30.

25. Lan M, Huang Y, Chen CY, Han F, Wu SX, Tian L, et al. Prognostic Value of Cervical Nodal Necrosis in Nasopharyngeal Carcinoma: Analysis of 1800 Patients with Positive Cervical Nodal Metastasis at MR Imaging. Radiology. 2015; 276: 536-44. 